\title{
Rare express saccades in elderly fallers
}

\author{
Q Yang' \\ TT Lê' \\ E Debay' \\ C Orssaud ${ }^{2}$ \\ G Magnier ${ }^{3}$ \\ Z Kapoula' \\ 'Groupe IRIS Vision and Motricité \\ Binoculaire, CNRS, Service \\ d'Ophtalmologie-ORL-Stomatologie; \\ ${ }^{2}$ Service d'Ophtalmologie, Hôpital \\ Européen Georges Pompidou, Paris, \\ France; ${ }^{3} \mathrm{Hôpital}$ de gériatrie Henry \\ Dunant, Paris, France
}

Objective: To examine horizontal saccades in elderly subjects with falling history; prior extensive screening was done to recruit subjects with falling history in the absence of pathology.

Methods: Twelve elderly with falling history were tested. Two testing conditions were used: the gap (fixation target extinguishes prior to target onset) and the overlap (fixation stays on after target onset) paradigms. Each condition was run at three viewing distances $-20 \mathrm{~cm}, 40 \mathrm{~cm}$, and $150 \mathrm{~cm}$, corresponding to convergence angle at $17.1^{\circ}, 8.6^{\circ}$, and $2.3^{\circ}$, respectively. Eye movements were recorded with the photoelectric IRIS (Skalar medical).

Results: (i) like in healthy elderly subjects, elderly with falling history produce shorter latencies in the gap paradigm than in the overlap paradigm; (ii) their latencies are shorter at near distances (20 and $40 \mathrm{~cm}$ ) relative to $150 \mathrm{~cm}$ for both paradigms; (iii) the novel result is that they fail to produce express latencies even in the conditions (near viewing distance and the gap task) known to promote high rates of express in adults $(25 \%)$ or in healthy elderly $(20 \%)$. Seven from the 10 healthy elderly produced express saccades at rates $>12 \%$, while 9 of the 12 older subjects with falling history showed no express saccades at all; the remaining 3 subjects showed low rates $<12 \%$.

Conclusion: The quasi paucity of express saccades could be due to the disequilibrium of complex cortical/subcortical networks needed for making express saccades. The results support models suggesting specific network for express saccades; missing of such optomotor reflex may go along with missing other reflexes as well increasing the chances of falling.

Keywords: elderly, falling, saccades, gap/overlap, express saccade

\section{Introduction}

Aging is associated with a decrease in the quality of balance regulation, in particular through dysfunctions of gaze control and postural stabilization, which results in an increased risk of a falling (Gauchard et al 2003). Gaze control is provided by two systems: gaze orientation by ocular saccades and gaze stabilization by reflexes and voluntary movements of vestibular and visual origins (Waespe and Henn 1987). The studies on healthy aged subjects with easy falling showed that the latency of saccades was prolonged (Yang and Kapoula 2006; Yang et al 2006a). Gauchard and colleagues (2003) reported that the reaction time and the accuracy of saccades were improved in a sporting group compared to a nonsporting group in elderly. They proposed that the practice of physical and sporting activities could improve every level of postural regulation and ocular movements of visual or vestibular origin to help reduce falls in elderly. However, no studies had been directly made on ocular saccades in elderly subjects with a history of falling.

Latency of saccades is the time between target displacement and the onset of the movement. Saccadic latency is a sensitive indicator of general response speed and reaction time towards a visual target to be centered or to be avoided. It is dependent on the state of fixation at the time of target appearance. Two types of paradigms have been introduced initially by Saslow (1967) and since then have been widely used to determine saccade latency under different states of visual attention: in the gap paradigm 
the fixation point disappears some time prior to target onset; in the overlap paradigm the fixation point remains illuminated during the target presentation. Latencies of saccades in the gap paradigm are generally reduced as compared to the overlap paradigm. This is called the gap effect (Saslow 1967) and could be due to attentional disengagement (Shepherd et al 1986; Fischer and Breitmeyer 1987; Fischer and Weber 1993; Hoffman and Subramaniam 1995), to advanced motor preparation (Pare and Munoz 1996) or to the disinhibition in the superior colliculus (SC) because of the offset of the fixation point (Reuter-Lorenz et al 1991; Findlay and Walker 1999). The second phenomenon is that subjects can generate an abundance of short-latency between $80 \mathrm{~ms}$ and $120 \mathrm{~ms}$ (Fischer and Ramsperger 1984) and express saccades in the gap paradigm. Express latencies are thought to be minimum conduct time (Munoz et al 1998).

A second parameter influencing latency is the viewing distance. Near distance also promotes shorter latency in both children and adults (Yang et al 2002). A recent study by our group using gap/overlap at far and near in healthy elderly showed that they were capable of producing express saccades in the gap paradigm and at near as frequently as young adults (Yang et al 2006a). The purpose of the present study is to examine elderly with falling history in the same battery of tests. The main questions addressed are the following: Do they show the gap effect? Can they produce express saccades in the gap paradigm and at near?

\section{Methods}

\section{Subjects}

Twelve subjects with the falling history (fell at least once last year) aged between 61 and 90 years (mean $76.8 \pm 9.2$ ) participated in this study. They were recruited from the geriatric services in the hospital after extensive examination including bedside eye-movement examination as described by Leigh and Zee (1999). These subjects were planned to participate in the intervention program for falls prevention organized by the hospital; the program includes the physical training, walking and equilibrium exercises, standing with eyes opened or closed, etc; a program run by physical therapists. No subject showed visual, neurological, psychiatric disorders, muscular-skeletal pathology, or received neurological/psychotropic drugs. All subjects had corrected visual acuity more than 5/10, ie, normal for their age. The cognitive performance as measured by Mini Mental State Examination (MMSE) which was better than 26 of 30 points, ie, a normal score (Folstein et al 1975). Binocular vision was assessed with the TNO (the Netherlands Organization of Applied Scientific Research) test of stereoacuity; all individual scores were normal, $60^{\circ}$ of arc or better. The investigation adhered to the tenets of the Declaration of Helsinki and was approved by the institutional human experimentation committee. Informed consent was obtained from all subjects after the nature of the procedure had been explained.

\section{Visual display}

The visual display consisted of LEDs (each LED on $2.9 \mathrm{~mm}$ ) placed at three isovergence circles: one at $20 \mathrm{~cm}$ from the subject, and the others at $40 \mathrm{~cm}$ and $150 \mathrm{~cm}$; on each circle three LEDs were used; one at the center and the others at $\pm 10^{\circ}$ (see Figure 1a). In a dark room, the subject was seated in an adapted chair with a chin and frontal rest. He/she viewed binocularly and faced the visual display of the LEDs.

\section{Fixation and oculomotor tasks: Gap and overlap conditions}

In the gap task, there was a time interval of $200 \mathrm{~ms}$ between the offset of the fixation point and the onset of the saccade target. The target LED was kept on for $1.5 \mathrm{sec}$ (Figure 1b). In the overlap task, the fixation point remained illuminated for $200 \mathrm{~ms}$ after the target LED appeared. The target stayed on also for another $1.5 \mathrm{sec}$ (Figure 1c). The total mean length of each trial was about 4 sec. In each block a target was presented at $10^{\circ}$, left or right from the center fixation; for each trial the fixation point and the peripheral target were at the same viewing distance $(20 \mathrm{~cm}, 40 \mathrm{~cm}$ or $150 \mathrm{~cm}$; see Figure 1a). Each block contained 60 trials (10 trials for each direction at each viewing distance) and lasted $4 \mathrm{~min}$. Each subject performed two blocks, one in the gap condition and another in the overlap condition. A calibration sequence was performed at the beginning and at the end of each block; the LED target stepped in a predictable way from center to left $5^{\circ}$, or $15^{\circ}$, then to right $5^{\circ}$, or $15^{\circ}$, each time returning to center.

\section{Eye movement recording}

Horizontal movements from both eyes were recorded simultaneously with the IRIS device (Skalar Medical BV, Delft, The Netherlands). Eye position signals were low-pass filtered with a cut-off-frequency of $200 \mathrm{~Hz}$ and digitized with a 12-bit analogue-to-digital converter and each channel was sampled at $500 \mathrm{~Hz}$. The head was always stabilized by a chinrest and/or a forehead rest.

\section{Data analysis}

From the two individual calibrated eye position signals we derived the conjugate signal [(left eye + right eye)/2]. 
a

\section{Horizontal saccades}

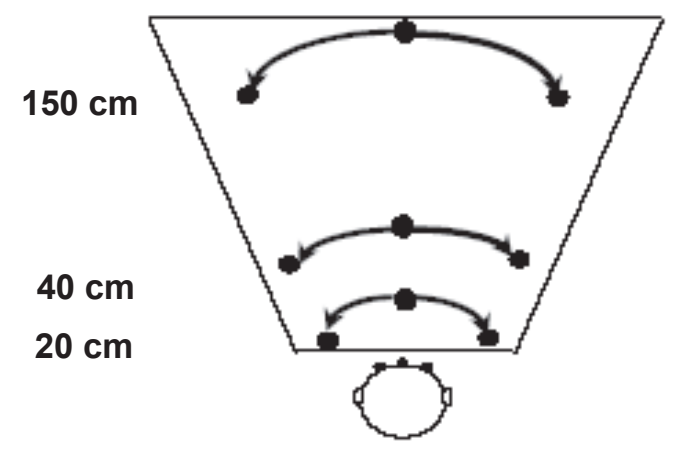

b

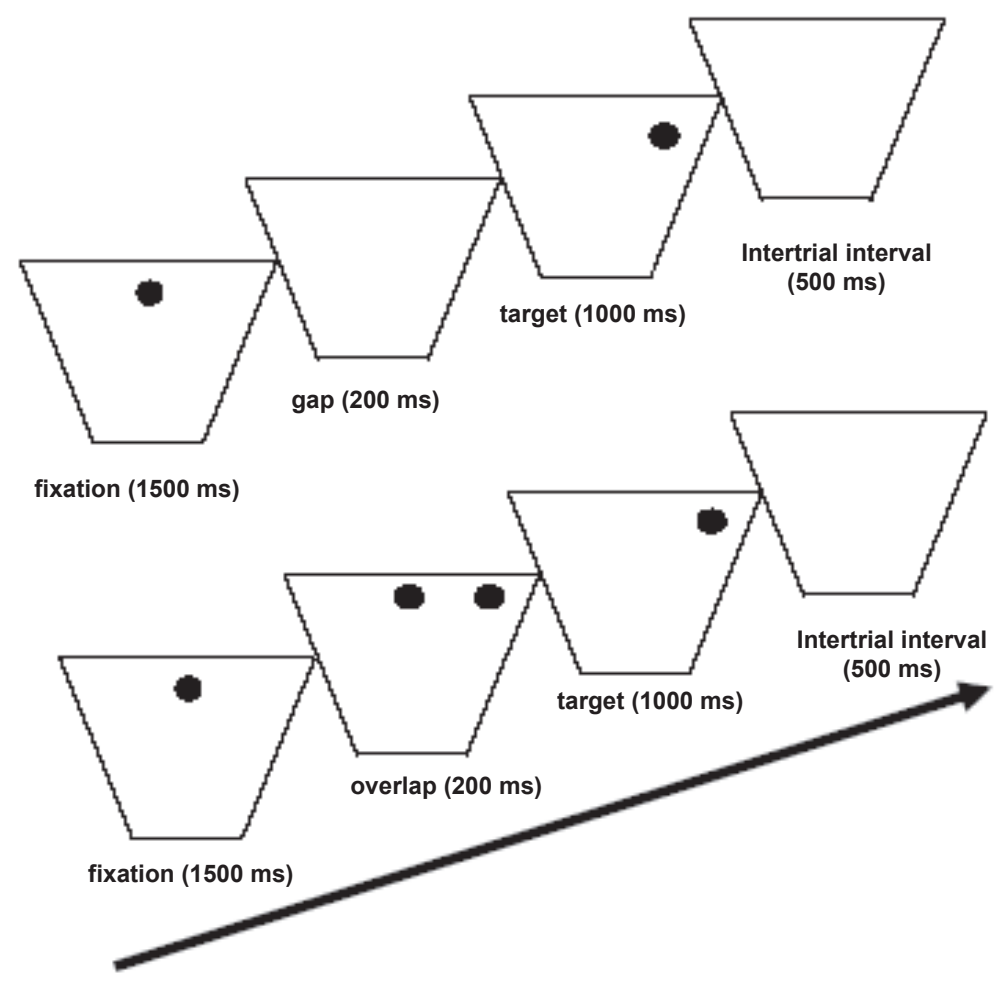

d

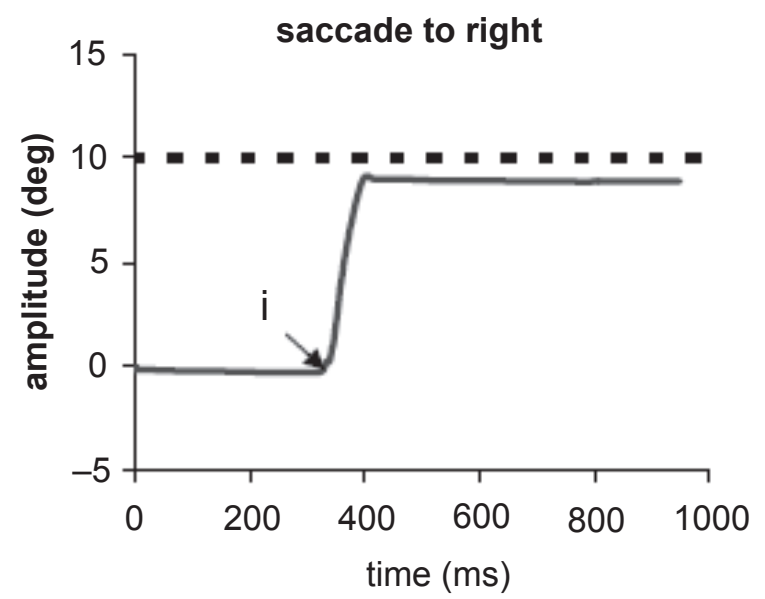

Figure I (a) Horizontal saccades elicited with the use of visual LEDs at $20 \mathrm{~cm}, 40 \mathrm{~cm}$, and $150 \mathrm{~cm}$ of the viewing distance from subjects' eyes. (b) The paradigm used for the gap condition, the central fixation point disappears $200 \mathrm{~ms}$ before the appearance of the eccentric target. (c) For the overlap condition, the central fixation point remains illuminated when an eccentric target appears. (d) Typical recordings of saccades are obtained by averaging the position signal of the two eyes (LE+RE)/2. The arrows at ' $i$ ' indicate the onset of movements. 
The onset of the saccade was defined as the time when conjugate eye velocity exceeded $30 \%$ sec (Figure $1 d$, point ' $i$ '). The process was performed automatically by the computer, and the verification was made by visual inspection of the individual eye position and velocity traces. For both gap and overlap tasks latency was measured as the time between target onset and saccade onset.

Eye movements in the wrong direction, with latency shorter than $80 \mathrm{~ms}$ (anticipation) or longer than $800 \mathrm{~ms}$, or contaminated by blinks were rejected. About $10 \%$ of trials had to be rejected using these criteria. We also evaluated the percentage of saccades with express type of short latency (80-120 ms).

A two-way ANOVA was performed on individual mean latency with the within subjects factors: the oculomotor task (gap, overlap) and the viewing distance (20, 40, and $150 \mathrm{~cm}$ ). One ANOVA was performed on individual mean latency for each condition with the between subjects: elderly fallers and healthy elderly as control subjects (Yang et al 2006a). For the percentage of express latency, the nonparametric test and Friedman and Wilcoxon test was used for within-subjects comparisons between different distances; the U-Mann-Whitney test was used for comparisons between elderly fallers and healthy elderly (control).

\section{Results}

\section{Mean latency: Gap and distance effects}

Figure 2 shows the individual mean latencies of saccades and their standard errors (SE) at different viewing distance $(20,40$, and $150 \mathrm{~cm})$ for the gap and the overlap conditions for healthy falling subjects. The two way ANOVA showed a statistically significant effect of the gap $\left(\mathrm{F}_{1,11}=55.56\right.$, $\mathrm{p}<0.001)$ and of the distance $\left(\mathrm{F}_{2,22}=6.25, \mathrm{p}<0.01\right)$. The gap effect, ie, shorter latency in the gap condition than in the overlap condition, is $106 \mathrm{~ms}$, and the distance effect, ie, shorter latency at near than at far, is $59 \mathrm{~ms}$ between $20 \mathrm{~cm}$ and $150 \mathrm{~cm}$, and $52 \mathrm{~ms}$ between $40 \mathrm{~cm}$ and $150 \mathrm{~cm}$. These
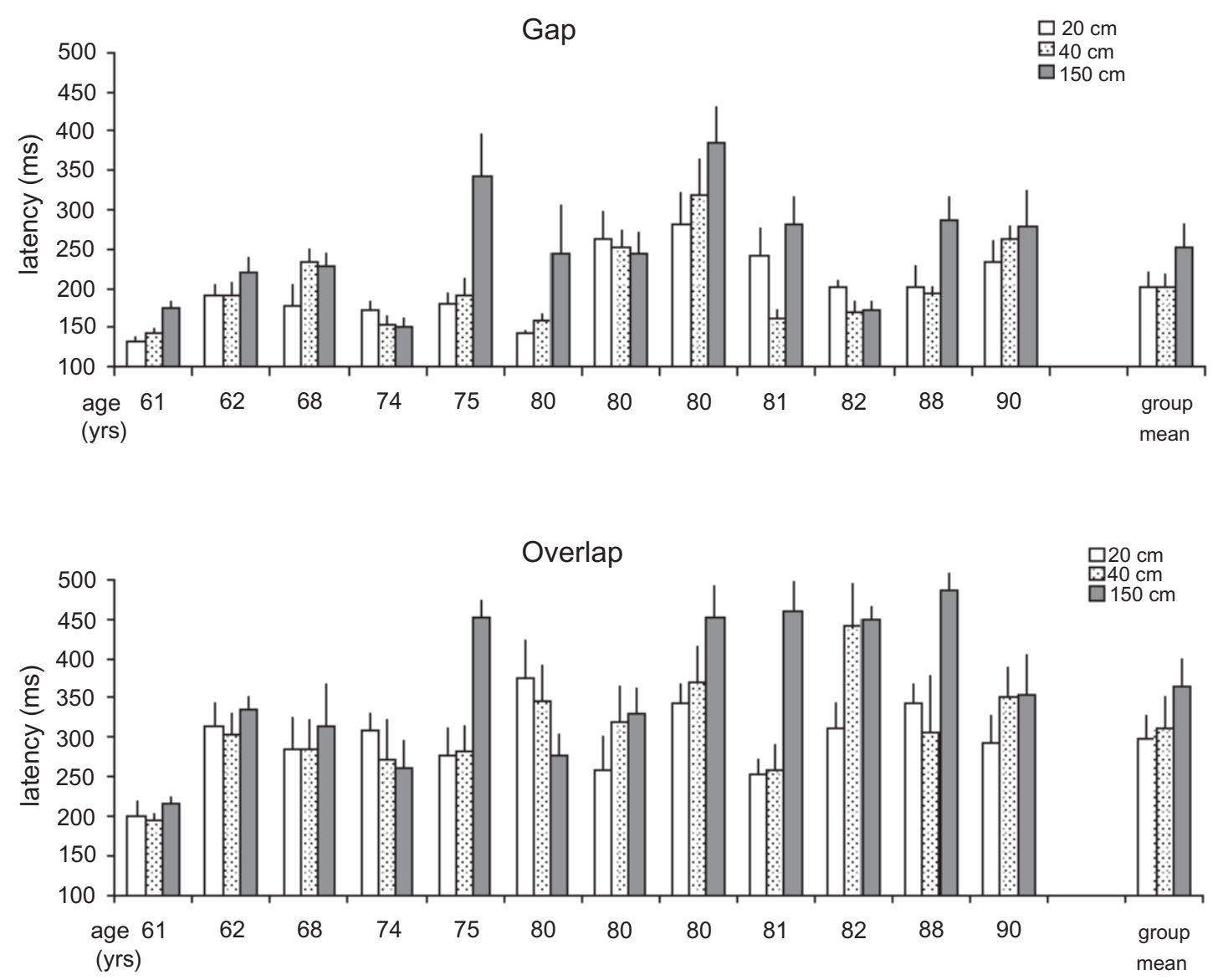

Figure 2 Individual and group mean latency with standard errors for saccades at $20 \mathrm{~cm}, 40 \mathrm{~cm}$, and I50 cm of viewing distances in the gap and the overlap conditions for elderly fallers. Latencies of saccades under the overlap conditions are longer than under the gap condition for each viewing distance. The distance effect, shorter latencies of saccades at near than at far, is significant between $20 \mathrm{~cm}$ and $150 \mathrm{~cm}$ and between $40 \mathrm{~cm}$ and I50 cm for both gap and overlap conditions (all p $<0.0 \mathrm{I}$ ), but not between $20 \mathrm{~cm}$ and $40 \mathrm{~cm}$. 
effects are similar to those obtained previously for aged control subjects (Yang et al 2006a). However, unlike elderly control subjects, falling subjects showed no interaction between the fixation task (gap/overlap) and the viewing distance $\left(\mathrm{F}_{2,22}=0.38, \mathrm{p}=0.69\right)$.

Further post-hoc comparisons showed that the gap effect was significant for all viewing distances (all $\mathrm{p}<0.01$ ). The distance effect was significant between $20 \mathrm{~cm}$ and $150 \mathrm{~cm}$ and between $40 \mathrm{~cm}$ and $150 \mathrm{~cm}$ for both gap and overlap conditions (all $\mathrm{p}<0.01$ ), but no significant difference was found between $20 \mathrm{~cm}$ and $40 \mathrm{~cm}(\mathrm{p}=0.93$ for the gap condition, or $\mathrm{p}=0.39$ for the overlap condition).

\section{Comparison with healthy control elderly}

One-way ANOVA showed longer latencies for elderly falling subjects than for elderly normal subjects (Yang et al 2006a) only in the gap condition at $20 \mathrm{~cm}\left(\mathrm{~F}_{1,20}=10.75, \mathrm{p}<0.01\right)$, but not in other conditions (all $\mathrm{p}>0.05$ ). Recall here that there are different types of saccadic latencies (Fischer et al 1997; Gezeck et al 1997), express type, $80-120 \mathrm{~ms}$; fast regular, 121-150 ms; and slow regular, 151-400 ms. A second oneway ANOVA was done for mean latencies excluding the express latency between 80 and $120 \mathrm{~ms}$ in the gap condition at $20 \mathrm{~cm}$. The results showed no statistically significant difference of latency between healthy elderly and falling subjects. Therefore, longer latency for elderly falling subjects in the gap condition at $20 \mathrm{~cm}$ could be related to the decrease of percentage of express, which will be presented below.

\section{Additional observation: Saccades to predictable targets}

Latencies of saccades to predicable targets (extracted from the calibration sequence; see Fixation and oculomotor tasks) were also analyzed. Mean latencies of saccades were $252 \pm 50 \mathrm{~ms}$ (mean $\pm \mathrm{SD}$ ) for elderly falling subjects and $268 \pm 58 \mathrm{~ms}$ for healthy control elderly. One-way ANOVA showed no significant difference of such latency between these two groups. In addition, there was no express latency for either group.

\section{Express type of latencies}

In the gap condition only 3 of the 12 falling subjects produced express latencies at $20 \mathrm{~cm}$. At distances of $40 \mathrm{~cm}$ and $150 \mathrm{~cm}$ only 1 subject produced express latencies, respectively. The remaining subjects showed no express latency. The group means of express percentages were only $2.1 \%, 0.6 \%$, and $0.4 \%$ at $20 \mathrm{~cm}, 40 \mathrm{~cm}$, and $150 \mathrm{~cm}$, respectively.

The distribution of the latencies in the gap condition at $20 \mathrm{~cm}$, which in controls is the most promoting condition for express saccades, is shown in Figure 3. Latencies with a few exceptions are all regular, ie, more than $120 \mathrm{~ms}$. The Friedman test used for within-subjects comparison showed no significant difference of express rates between different distances for falling subjects in the gap condition $(\mathrm{F}=3.80$, $p>0.05)$. The overlap condition produced express type of latency rarely.

\section{Gap at $20 \mathrm{~cm}$}

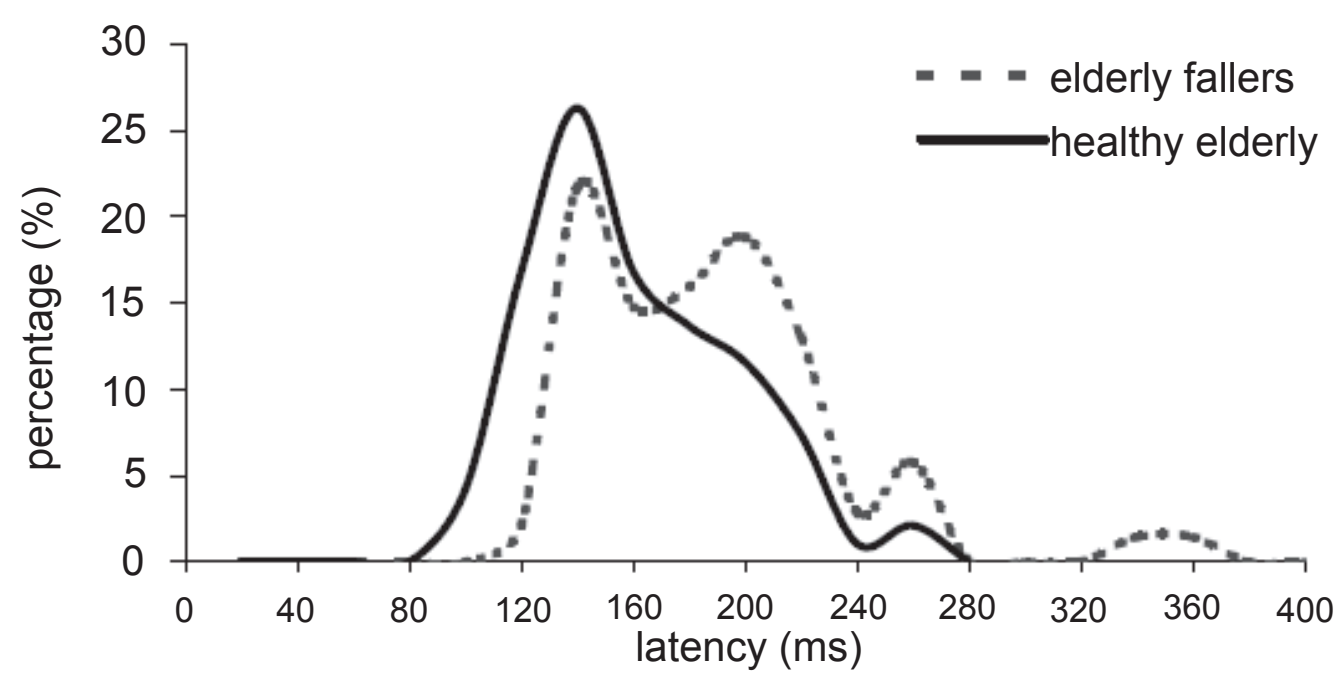

Figure 3 The latencies distribution for all elderly falling subjects in the gap condition at $20 \mathrm{~cm}$; the percentage of express saccades (between 80 and I20 ms) is only $2.1 \%$ in elderly fallers and less than $20 \%$ in healthy elderly. 


\section{Comparison with healthy control elderly}

The percentage of express latencies for control subjects was $20 \%, 5 \%$, and $1 \%$ in the gap condition at $20 \mathrm{~cm}, 40 \mathrm{~cm}$ and $150 \mathrm{~cm}$, respectively. At near $(20 \mathrm{~cm})$ the percentage for control subjects is significantly higher than that observed for falling subjects ( $U$-Mann-Whitney test, $U=25, p<0.05$; see Table 1), but not for other distances $(\mathrm{P}>0.05)$.

\section{Discussion}

The main results of this study are: (i) like in control subjects, elderly with falling history produce shorter latencies in the gap paradigm than in the overlap paradigm; (ii) latencies of saccades at near are shorter than those at far; this is the case for both tasks (gap and overlap); (iii) relative to healthy elderly, the large majority of subjects with falling history fail to produce express saccades even in the most favorable conditions, eg, in the gap task and at near.

\section{The gap effect and the distance effect}

The gap effect of horizontal saccades, ie, shorter latencies in the gap paradigm than in the overlap paradigm, was similar for healthy elderly (107 ms) and young adults (91 ms, (Yang et al 2006a). Moreover, the percentages of latency decrease in the gap condition (overlap-gap/overlap) were $37.2 \%$ and $37 \%$ for young and healthy elderly subjects, respectively. In this study for elderly subjects with falling history, such decrease was similar at $32.7 \%$. In general, the gap effect refers to the latency advantage of removing the fixation point before the onset of a target (Pratt et al 2006). Although the gap effect has been examined in many studies since the first report by

Table I Individual percentage of express latency under gap condition (at $20 \mathrm{~cm}$ )

\begin{tabular}{lll}
\hline Normal elderly & falling elderly \\
\hline 0.0 & 5.0 \\
25.0 & 0.0 \\
0.0 & 0.0 \\
12.5 & 0.0 \\
22.5 & 7.5 \\
37.5 & 0.0 \\
20.0 & 0.0 \\
0.0 & 12.5 \\
& 12.5 & 0.0 \\
70 & 0.0 \\
& & 0.0 \\
mean & 0.0 \\
\hline
\end{tabular}

Saslow (1967), the exact mechanism for producing such effect is still controversial. Fischer and colleagues (1987, 1993) thought that the gap effect was due to attentional disengagement that was facilitated by the removal of the fixation point. However, Kingstone and Klein (1993) found a large gap effect with the offset of a fixated object but a much smaller gap effect when an attended but not fixated object was offset, suggesting a limited role for attention in the gap effect. An alternative explanation for the gap effect suggested that the offset of the fixation point produces disinhibition in the SC (Reuter-Lorenz et al 1991). Whatever the mechanism is, our results suggest that the mechanism is operating similarly in elderly subjects with falling history as in both healthy young and old subjects did. It seems to be a robust mechanism independent of age and other functional problems leading to fall.

The distance effect of horizontal saccades, ie, shorter latency at close than at far, was found for both children and adults (Yang et al 2002). Recently, we reported such distance effect existed in healthy elderly but in the gap condition only (Yang et al 2006a). We suggested that the distance effect could be sensory (large angle size and luminance of LEDs at close), oculomotor (high convergence relaxation at close), attentional (facilitation of disengagement of visual attention when the eyes converge at close), or any combination of these factors (Yang et al 2002). In another study dealing with vertical saccades the distance effect was not found (Yang and Kapoula 2006). Thus, the distance effect clearly involves the oculomotor factors. The present study shows a distance effect for both tasks: latencies increase with distance increase. Note that healthy elderly subjects showed the distance effect only in the gap condition and we have argued that the distance effect was somehow hidden in the overlap condition. For subjects with falling history latencies of saccades at $150 \mathrm{~cm}$ becomes longer ( $36 \mathrm{~ms}$ relative to control subjects but not reach significance) and such prolongation could lead to significant distance effect (longer latency for saccades at far).

\section{Quasi paucity of express saccades}

Express saccades were first observed in monkeys (Fischer and Boch 1983) and then in humans (Fischer et al 1984). The ability to produce express saccades is variable in normal humans; the large majority of humans produce express saccades at rates less than $30 \%$ in the gap paradigm (Fischer and Weber 1993); a few subjects called 'express-saccade makers' can produce unusually high numbers of express saccades even in the overlap task (Biscaldi et al 1996; Kimmig et al 2002). 
Nearness and the gap paradigm are shown to be two aspects promoting express saccades (Yang et al 2006b). In this previous study we showed that 8 of the 9 young subjects produced express saccades at rates between $10 \%$ and $30 \%$ except one subject at $100 \%$. Among the 10 healthy elderly examined in the same study 7 showed express saccades at rates $>12 \%$; one subject made express saccades up to $70 \%$ (see Table 1 ). In the present study we used the same promoting conditions (gap paradigm at $20 \mathrm{~cm}$ ) but 9 of the 12 elderly with falling history showed no express saccades at al; the remaining 3 subjects produced express saccades at moderate rates $5 \%, 7.5 \%$, and $12.5 \%$. Although a larger number of falling subjects needs to be investigated such frequent paucity of express saccades has theoretical and clinical value. High rate of express saccades found in dyslexic children has been explained as a sign of a weak fixation function, which normally inhibits the generation of reflexive saccades (Biscaldi et al 1996, 1998). The physiological significance of quasi absence of express latency is less clear. This result will be discussed in the context of existing models and data for generating express saccades.

Many models attempt to explain the production of express saccades. Majority of them admit the idea of a specific short circuit for the initiation of express saccades relative to regular saccades. For example, Fischer and colleagues (1993) proposed three parallel pathways, all of them including SC, frontal eye field (FEF), and posterior parietal cortex (PPC) for generating express, fast-regular or slow-regular saccades; the switching between pathways can be modulated by peripheral attention. Pierrot-Deseilligny and colleagues (1993) proposed a retina-parietal-superior colliculus circuit for triggering express saccades; Schiller and Tehovnik (2005) proposed a similar model. This was compatible with studies on patients with parietal lesions who showed decrease of express saccades (Braun et al 1992). Brown and colleagues (2004) suggested that the FEF is involved in the triggering of all types of saccades: although the production of express saccades is controlled by a subcortical circuit, eg, SC short circuit, it also depends on the quality of general cerebral function; namely frontal function which is necessary to learn to produce different strategies of saccades, reflexive-express versus voluntary. The authors emphasized the laminar structure of FEF and its interaction with the basal ganglia, thalamus, SC, inferotemporal and parietal cortex to perform different types of saccades including express saccades. This was in line with physiological studies in which FEF neurons in monkeys have been shown to increase their discharge rate in response to the disappearance of the fixation point during the gap (Dias and Bruce 1994; Everling and Munoz 2000).
Isa and Kobayashi (2004) suggested that superficial layer of SC mediate the express saccades (short-loop) while regular saccades are mediated by cerebral cortex (long-loop). The SC route for express saccades has been supported by Schiller and colleagues (1987), who showed that SC ablation obviated express saccades. The model of Isa and Kobayashi (2004) did not exclude that cortical areas were also involved in the production of express saccades but in different ways.

Our previous results in healthy elderly subjects indicated that percentages of express saccades were as high as for young subjects (Yang et al 2006a). Therefore, fewer or quasi absent express saccades in elderly falling subjects suggests that the circuit for triggering express saccades or the more global cortical circuit needed learn to make express saccades could be impaired. Particularly, reduced express saccades could be due to hypofunction of different areas believed to be involved in the triggering of express saccades. Recent anatomical studies confirmed that the ventral intraparietal area in monkeys (corresponding PPC in humans) can also receive direct projections from vestibular areas and thus is part of a cortical vestibular networks (Lewis and Van Essen 2000; Klam and Graf 2003). More over, the SC has been identified as the primary brainstem center controlling the orienting movement, ie, head, eyes and body in a wellcoordinated manner. Its output signal is transmitted to neck motoneurons (Isa and Sasaki 2002). Corneil and colleagues (2004) demonstrated that express saccades are accompanied by activation of ipsilateral neck muscles and inhibition of contralateral neck muscles relative to the side of target presentation. Thus, the PPC and the SC are important not only for express saccades but also for the equilibrium. In general, we could look at the express saccades as an optomotor reflex mediated by the colliculus, when allowed by cortical mechanisms. The missing of such optomotor reflex may go along with missing other reflexes as well increasing the chances of falling. Perhaps, absence or rare express saccade could be an early sign for falling in elderly, which is important for the early diagnosis and prevention of fall in elderly.

Finally, one could note that in laboratory conditions the gap eliciting express saccades uncovers ecological mechanisms. Indeed, in every day life our fixation can be instantaneously released and saccades to surrounding targets could be express. Fast saccade reaction time could be useful in everyday life, for example, during locomotion for avoiding the obstacles. Perhaps in persons with falling history the capacity to initiate fast saccades being diminished their ability to detect obstacles rapidly and avoid them is also decreased. 


\section{Acknowledgments}

Q.Yang was supported by European Union (QLK6-CT2002-00151: EUROKINESIS) and CNRS/CTI, Handicap contract. The authors thank the reviewers for the good suggestions for discussion. The authors report no conflicts of interest in this work.

\section{Reference}

Biscaldi M, Fischer B, Stuhr V. 1996. Human express saccade makers are impaired at suppressing visually evoked saccades. J Neurophysiol, 76:199-214.

Biscaldi M, Gezeck S, Stuhr V. 1998. Poor saccadic control correlates with dyslexia. Neuropsychologia, 36:1189-202.

Braun D, Weber H, Mergner T, et al. 1992. Saccadic reaction times in patients with frontal and parietal lesions. Brain, 115(Pt 5):1359-86.

Brown JW, Bullock D, Grossberg S. 2004. How laminar frontal cortex and basal ganglia circuits interact to control planned and reactive saccades. Neural Netw, 17:471-510.

Corneil BD, Olivier E, Munoz DP. 2004. Visual responses on neck muscles reveal selective gating that prevents express saccades. Neuron, 42:831-41.

Dias EC, Bruce CJ. 1994. Physiological correlate of fixation disengagement in the primate's frontal eye field. J Neurophysiol, 72:2532-7.

Everling S, Munoz DP. 2000. Neuronal correlates for preparatory set associated with pro-saccades and anti-saccades in the primate frontal eye field. J Neurosci, 20:387-400.

Findlay JM, Walker R. 1999. A model of saccade generation based on parallel processing and competitive inhibition. Behav Brain Sci, 22:661-74; discussion 674-721.

Fischer B, Boch R. 1983. Saccadic eye movements after extremely short reaction times in the monkey. Brain Res, 260:21-6.

Fischer B, Boch R, Ramsperger E. 1984. Express-saccades of the monkey: effect of daily training on probability of occurrence and reaction time. Exp Brain Res, 55:232-42.

Fischer B, Breitmeyer B. 1987. Mechanisms of visual attention revealed by saccadic eye movements. Neuropsychologia, 25:73-83.

Fischer B, Gezeck S, Hartnegg K. 1997. The analysis of saccadic eye movements from gap and overlap paradigms. Brain Res Brain Res Protoc, 2:47-52.

Fischer B, Ramsperger E. 1984 Human express saccades: extremely short reaction times of goal directed eye movements. Exp Brain Res, 57:191-5.

Fischer B, Weber H. 1993. Express saccades and visual attention. Behav Brain Sci, 16:553-610.

Folstein MF, Folstein SE, McHugh PR. 1975 "Mini-mental state". A practical method for grading the cognitive state of patients for the clinician. J Psychiatr Res, 12:189-98.

Gauchard GC, Gangloff P, Jeandel C, et al. 2003. Physical activity improves gaze and posture control in the elderly. Neurosci Res, 45:409-17.

Gezeck S, Fischer B, Timmer J. 1997. Saccadic reaction times: a statistical analysis of multimodal distributions. Vision Res, 37:2119-31.

Hoffman JE, Subramaniam B. 1995. The role of visual attention in saccadic eye movements. Percept Psychophys, 57:787-95.

Isa T, Kobayashi Y. 2004. Switching between cortical and subcortical sensorimotor pathways. Prog Brain Res, 143:299-305.
Isa T, Sasaki S. 2002. Brainstem control of head movements during orienting; organization of the premotor circuits. Prog Neurobiol, 66:205-41.

Kimmig H, Biscaldi M, Mutter J, et al. 2002. The initiation of smooth pursuit eye movements and saccades in normal subjects and in "express-saccade makers". Exp Brain Res, 144:373-84.

Kingstone A, Klein RM. 1993. Visual offsets facilitate saccadic latency: does predisengagement of visuospatial attention mediate this gap effect? J Exp Psychol Hum Percept Perform, 19:1251-65.

Klam F, Graf W. 2003. Vestibular signals of posterior parietal cortex neurons during active and passive head movements in macaque monkeys. Ann NY Acad Sci, 1004:271-82.

Leigh RJ, Zee DS. 1999. The neurology of eye movements. New York: Oxford University Press.

Lewis JW, Van Essen DC. 2000. Mapping of architectonic subdivisions in the macaque monkey, with emphasis on parieto-occipital cortex. J Comp Neurol, 428:79-111.

Munoz DP, Broughton JR, Goldring JE, et al. 1998. Age-related performance of human subjects on saccadic eye movement tasks. Exp Brain Res, 121:391-400.

Pare M, Munoz DP. 1996. Saccadic reaction time in the monkey: advanced preparation of oculomotor programs is primarily responsible for express saccade occurrence. J Neurophysiol, 76:3666-81.

Pierrot-Deseilligny C, Israel I, Berthoz A, et al. 1993. Role of the different frontal lobe areas in the control of the horizontal component of memoryguided saccades in man. Exp Brain Res, 95:166-71.

Pratt J, Lajonchere CM, Abrams RA (2006) Attentional modulation of the gap effect. Vision Res, 46:2602-7.

Reuter-Lorenz PA, Hughes HC, Fendrich R. 1991. The reduction of saccadic latency by prior offset of the fixation point: an analysis of the gap effect. Percept Psychophys, 49:167-75.

Saslow MG. 1967. Effects of components of displacement-step stimuli upon latency for saccadic eye movement. J Opt Soc Am, 57:1024-9.

Schiller PH, Sandell JH, Maunsell JH. 1987. The effect of frontal eye field and superior colliculus lesions on saccadic latencies in the rhesus monkey. J Neurophysiol, 57:1033-49.

Schiller PH, Tehovnik EJ. 2005. Neural mechanisms underlying target selection with saccadic eye movements. Prog Brain Res, 149:157-71.

Shepherd M, Findlay JM, Hockey RJ. 1986. The relationship between eye movements and spatial attention. $Q J$ Exp Psychol A, 38:475-91.

Waespe W, Henn V. 1987. Gaze stabilization in the primate. The interaction of the vestibulo-ocular reflex, optokinetic nystagmus, and smooth pursuit. Rev Physiol Biochem Pharmacol, 106:37-125.

Weerdesteyn V, Rijken H, Geurts AC, et al. 2006. A five-week exercise program can reduce falls and improve obstacle avoidance in the elderly. Gerontology, 52:131-41.

Yang Q, Bucci MP, Kapoula Z. 2002. The latency of saccades, vergence, and combined eye movements in children and in adults. Invest Ophthalmol Vis Sci, 43:2939-49.

Yang Q, Kapoula Z. 2006. The control of vertical saccades in aged subjects. Exp Brain Res, 171:67-77.

Yang Q, Kapoula Z, Debay E, et al. 2006a. Prolongation of latency of horizontal saccades in elderly is distance and task specific. Vision Res, 46:751-9.

Yang Q, Kapoula Z, Debay E, et al. 2006b. Prolongation of latency of horizontal saccades in elderly is distance and task specific. Vision Res, 46:751-9. 